\title{
Let-7 represses Nr6a1 and a mid-gestation developmental program in adult fibroblasts
}

\author{
Allan M. Gurtan, ${ }^{1}$ Arvind Ravi, ${ }^{2}$ Peter B. Rahl, ${ }^{3}$ Andrew D. Bosson, ${ }^{1,4}$ Courtney K. JnBaptiste, ${ }^{1,4}$ \\ Arjun Bhutkar, ${ }^{1}$ Charles A. Whittaker, ${ }^{1}$ Richard A. Young, ${ }^{3}$ and Phillip A. Sharp ${ }^{1,4,5}$ \\ ${ }^{1}$ David H. Koch Institute for Integrative Cancer Research, Cambridge, Massachusetts 02139, USA; ${ }^{2}$ Harvard-Massachussets \\ Institute of Technology Health Sciences and Technology Program, Cambridge, Massachusetts 02139, USA; ${ }^{3}$ Whitehead Institute \\ for Biomedical Research, Cambridge, Massachusetts 02142, USA; ${ }^{4}$ Department of Biology, Massachusetts Institute of \\ Technology, Cambridge, Massachusetts 02139, USA
}

\begin{abstract}
MicroRNAs (miRNAs) are critical to proliferation, differentiation, and development. Here, we characterize gene expression in murine Dicer-null adult mesenchymal stem cell lines, a fibroblast cell type. Loss of Dicer leads to derepression of let-7 targets at levels that exceed 10-fold to 100-fold with increases in transcription. Direct and indirect targets of this miRNA belong to a mid-gestation embryonic program that encompasses known oncofetal genes as well as oncogenes not previously associated with an embryonic state. Surprisingly, this mid-gestation program represents a distinct period that occurs between the pluripotent state of the inner cell mass at embryonic day 3.5 (E3.5) and the induction of let-7 upon differentiation at E10.5. Within this mid-gestation program, we characterize the let-7 target Nr6a1, an embryonic transcriptional repressor that regulates gene expression in adult fibroblasts following miRNA loss. In total, let-7 is required for the continual suppression of embryonic gene expression in adult cells, a mechanism that may underlie its tumor-suppressive function.
\end{abstract}

[Keywords: Dicer; miRNAs; let-7; embryo; cancer; oncofetal]

Supplemental material is available for this article.

Received February 2, 2013; revised version accepted March 27, 2013.

MicroRNAs (miRNAs) are $\sim 22$-nucleotide (nt) RNAs that regulate various processes (Gurtan and Sharp 2013), including organismal development (Bernstein et al. 2003; Harfe et al. 2005), proliferation (Johnson et al. 2005, 2007), and apoptosis (Brennecke et al. 2003; Ghildiyal and Zamore 2009). The seed sequence (positions 2-7) of a miRNA binds the 3' untranslated region (UTR) of a target mRNA, leading to mRNA destabilization and/or translational inhibition (Ghildiyal and Zamore 2009). A single miRNA typically accounts for approximately twofold repression of a target. Due to this mild activity, miRNAs are considered "fine-tuners" of gene expression that act in concert with other classes of regulators (Bartel and Chen 2004). Accordingly, miRNAs shape gene expression by participating in circuits with transcription factors, modulating the kinetics of gene activation or repression, and providing a buffer against perturbation (Herranz and Cohen 2010; Ebert and Sharp 2012).

In vivo, miRNAs are critical to organismal development and survival, while in vitro, miRNAs are dispensable for viability of certain cell lines. In the whole animal, genetic ablation of Dicer1 or $\operatorname{Dgcr} 8$, required for miRNA biogenesis, leads to early embryonic lethality and severe differentiation defects (Bernstein et al. 2003; Wang et al.

${ }^{5}$ Corresponding author

E-mail sharppa@mit.edu

Article is online at http://www.genesdev.org/cgi/doi/10.1101/gad.215376.113.
2007). Similarly, tissue-specific loss of miRNAs during differentiation leads to dysfunction or death in a variety of tissues, including myocytes (O'Rourke et al. 2007), lymphocytes (Muljo et al. 2005; Koralov et al. 2008), and epidermal cells (Andl et al. 2006; Yi et al. 2006). However, global loss of miRNAs is readily tolerated in vitro in various cell types, including embryonic stem cells (ESCs) (Kanellopoulou et al. 2005; Murchison et al. 2005; Calabrese et al. 2007; Wang et al. 2007), murine embryonic fibroblasts (Shapiro et al. 2012), sarcoma cells (Ravi et al. 2012), and immortalized adult mesenchymal stem cells (MSCs) (Gurtan et al. 2012; Ravi et al. 2012).

miRNA-deficient cell lines have been a useful resource for dissecting the role of miRNAs in gene expression programs and cellular phenotypes. Studies of Dicer1- and Dgcr8-knockout ESCs have elucidated the roles of the ESC-specific miR-290 family in regulating pluripotency networks in conjunction with transcription factors such as Oct4 (Kanellopoulou et al. 2005; Wang et al. 2007; Marson et al. 2008; Melton et al. 2010). The characterization of these miRNA-deficient ESCs in comparison with their wild-type counterparts or upon add-back of synthetic miRNAs has provided global signatures of activity and furthermore delineated the contribution of a single miRNA family to these signatures.

Few in vitro studies analogous to those in miRNA-deficient ESCs have been reported for somatic cells, which represent a counterpoint to the pluripotent state. The 
existing somatic in vitro studies have yielded significant insight into the activity of miRNAs in differentiated cell types (Dugas et al. 2010). Many somatic cells predominantly express let-7, a highly conserved miRNA known for its role in opposing self-renewal programs regulated by miR-290 (Melton et al. 2010). In multiple species, ranging from nematodes to mammals, mature let-7 becomes expressed in the developing embryo (Nimmo and Slack 2009; Ambros 2011). In nematodes, loss of the let-7 family results in the reiteration of larval stages. Let-7 is similarly thought to regulate developmental timing in mammals (Schulman et al. 2005). Exogenous expression of mature let-7 in ESCs in vitro represses pluripotency programs and antagonizes the activity of the miR-290 family (Melton et al. 2010). In vivo, however, the dispersion of let-7 genes across multiple genomic loci has confounded its genetic analysis in mammalian development.

In the adult, mature let-7 persists at high levels and functions as a tumor suppressor (Boyerinas et al. 2010; Trang et al. 2010). Many targets of let-7 are oncogenes, and reduction of let-7 levels is frequently observed in cancer. A small subset of let-7 targets falls into a conceptual class of genes termed "oncofetal" because of their expression profile (Boyerinas et al. 2008; Gurtan and Sharp 2013): highly expressed in the embryo, inactive in most adult tissues, and re-expressed in tumors. The only oncofetal targets of let-7 characterized thus far have been the RNA-binding families Lin-28a/b and Igf2bp1-3 as well as the nonhistone chromatin factor Hmga2 (Lee and Dutta 2007; Mayr et al. 2007; Boyerinas et al. 2008; Mayr and Bartel 2009). These oncofetal targets regulate proliferation and growth. Transgenic mice that express elevated levels of Lin-28 are larger than control littermates (Zhu et al. 2010, 2011), while knockouts of Hmga2 (Zhou et al. 1995) or Igf2bp1 (Hansen et al. 2004) exhibit dwarf phenotypes. Transgenic overexpression of Hmga2 (Fedele et al. 2006; Zaidi et al. 2006) or Igf2bp 1 (Tessier et al. 2004) results in tumorigenesis. Given the large number of predicted but uncharacterized targets of let-7, additional genes may populate networks typified by known oncofetal genes.

Here, we exploit a murine Dicer-null somatic cell line to identify gene expression programs regulated by let- 7 specifically and somatic miRNAs generally in the context of the adult. Deletion of Dicer in adult MSCs, a fibroblast cell type that abundantly expresses let-7, results in specific, transcriptionally reinforced changes in gene expression, including global derepression of miRNA targets. Dicer-null MSCs largely retain their cellular identity but, within this landscape of mesenchymal gene expression, activate a discrete let-7-targeted mid-gestation developmental program that includes known oncofetal genes as well as oncogenes not previously associated with an embryonic state. Within this mid-gestation program, we characterize the let-7 target Nr6a1, an embryonic transcriptional repressor that contributes to gene expression changes in adult fibroblasts following miRNA loss. In total, we report that somatic miRNAs such as let-7 are required for the continual suppression of embryonic gene expression in adult cells, a mechanism that may underlie their tumor-suppressive functions.

\section{Results}

Dicer knockout adult fibroblasts exhibit specific changes in gene expression

To understand the role of miRNAs in regulating gene expression in somatic cells, we performed expression profiling in a recently described model of Dicer loss. Specifically, we used immortalized clonal lines of Dicer1 $1^{f / f}$ (Dicer wild-type) and Dicer1 ${ }^{-1-}$ (Dicer knockout) MSCs, a fibroblast cell type present in adult bone marrow (Ravi et al. 2012). To establish a baseline of miRNA expression in these fibroblast cell lines, we performed small RNA sequencing (small RNA-seq) (Supplemental Table S1). In Dicer wild-type MSCs, the five most abundant miRNAs were miR-22 (17\%), let-7c (16\%), let-7b (9\%), miR-16 $(7 \%)$, and miR-145 (6\%). Collapsing miRNAs by seeds, the let- 7 family comprised $37 \%$ of all miRNA reads, consistent with published observations of let-7 predominance in somatic tissues (Marson et al. 2008). This expression profile is comparable with previous studies in these cells (Gurtan et al. 2012) and closely resembles the profile observed in Kras ${ }^{G 12 D}$;Trp53 ${ }^{-/-}$;Dicer $1^{f /-}$ sarcoma cells (Ravi et al. 2012). Mature miRNAs constituted $55 \%$ of total small RNA reads in Dicer wild-type MSCs but only $0.5 \%$ of total reads in Dicer knockout MSCs, similar to the degree of loss observed in sarcoma cells. Per cell, most miRNAs were present at fewer than one copy in Dicer knockout MSCs (Supplemental Fig. S1A). Members of the let-7 family were reduced from thousands of copies per cell in Dicer wild-type MSCs to $<10$ copies per cell in Dicer knockout MSCs (Supplemental Fig. S1A).

Next, we characterized the consequences of miRNA loss on gene expression. By exon microarray, we observed specific gene expression differences between Dicer wildtype and knockout MSCs (Fig. 1A; Supplemental Table S2), with unsupervised hierarchical clustering distinguishing the cells by genotype (Supplemental Fig. S1B). Two-hundred-seventeen genes were differentially expressed (adjusted $P$-value $\leq 0.1$ ) (Fig. 1A). The median change for both up-regulated and down-regulated genes was approximately threefold (Fig. 1A, gray dashed lines) with a maximum change of $\sim 50$-fold.

Conserved targets of conserved miRNAs, as predicted by TargetScan, were largely up-regulated in Dicer knockout MSCs (Fig. 1B), particularly for let-7, miR-199, and miR-15, which are three of the most highly expressed miRNA families in Dicer wild-type MSCs. We also observed Dicer loss-dependent up-regulation of targets of miR-202, which is not expressed in Dicer wild-type MSCs but shares a hexamer seed match with let-7. By gene set enrichment analysis (GSEA), we characterized the enrichment of miRNA target sites in the 3' UTRs of differentially expressed genes. Among genes up-regulated in Dicer knockout MSCs, 37 miRNA motifs were enriched at a false discovery rate (FDR) $q$-value $\leq 0.1$, including let- 7 $($ FDR $q$-value $=0.01)$ (Supplemental Fig. S1C), miR-199 $($ FDR $q$-value $=0.02)$, and miR-15 (FDR $q$-value $=0.03)$. Among genes down-regulated in Dicer knockout MSCs, only two miRNA seed-match motifs (miR-339 and miR-517) 
A

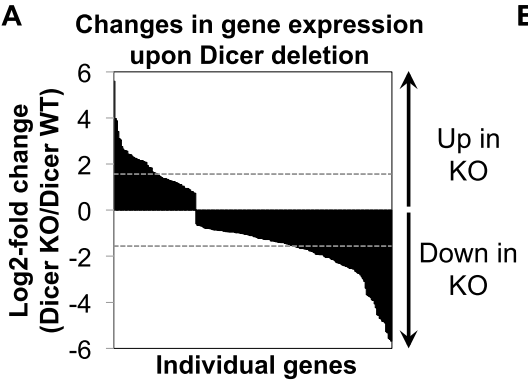

C

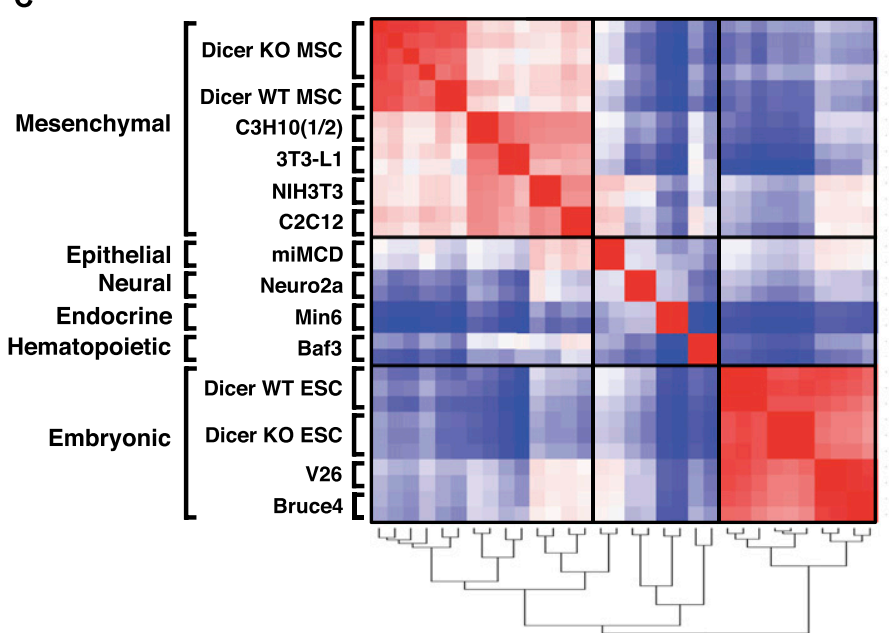

B

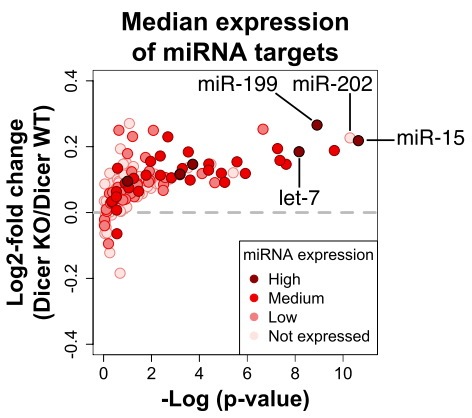

$\Pi^{1.00}$

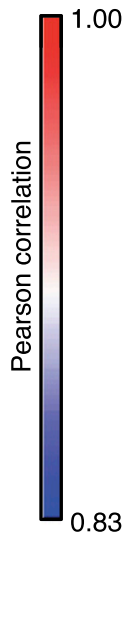

Figure 1. Loss of Dicer in adult MSCs results in specific gene expression changes. $(A)$ Waterfall plot of genes expressed differentially by exon microarray at an adjusted $P$-value $\leq 0.1$. Two-hundredseventeen genes are shown. Dashed gray lines indicate median up-regulation (threefold) and downregulation (threefold). Two Dicer $1^{f / f}$ (Dicer wildtype [WT]) and four Dicer1 ${ }^{-1-}$ (Dicer knockout [KO]) clones were evaluated for gene expression changes. $(B)$ Scatter plot of the median change in expression of miRNA targets relative to control gene sets matched for 3' UTR length, GC content, and expression in Dicer wild-type MSCs. Each point represents conserved targets of a single miRNA seed family. miRNA expression is based on the small RNA-seq data reported in this study. The $P$-value, whose negative $\log _{10}$ is shown on the $X$-axis, was calculated by Wilcoxon rank-sum test. $(C)$ Crosscorrelation analysis of Dicer wild-type and knockout MSCs versus a cell line panel based on total gene expression by exon microarray. The gene expression of Dicer wild-type and knockout ESCs was published previously (Leung et al. 2011). The gene expression of all other murine cell lines is publicly available from Novartis BioGPS (Wu et al. 2009, 2013). See also Supplemental Figure S1 and Supplemental Tables S1 and S2. were identified at an FDR $q$-value $\leq 0.1$. Thus, Dicer loss resulted in derepression of miRNA targets in MSCs.

\section{Dicer knockout fibroblasts retain mesenchymal cell identity}

Tissue-specific deletion of Dicer in vivo during development often results in differentiation defects or lethality, suggesting that miRNAs are critical in establishing or maintaining cellular identity. Therefore, we determined whether gene expression changes in Dicer knockout MSCs in vitro reflected a gross change in cellular identity. We compared the full gene expression profiles of Dicer wild-type and knockout MSCs with those of a diverse array of cell types ranging from ESCs to neural cells $(\mathrm{Wu}$ et al. 2009, 2013). Cells generally clustered by cell type, and the profile of Dicer wild-type MSCs correlated most closely with mesenchymal cells such as 3T3-L1 and C3H10(1/2) cells (Fig. 1C). Dicer knockout MSCs remained mesenchymal, correlating most closely with Dicer wildtype MSCs. Thus, MSCs retain their identity independent of miRNAs.

To test whether this observation could be extended to another cell type, we also analyzed Dicer wild-type and knockout ESCs generated from the same Dicer-conditional mouse model (Leung et al. 2011). Both Dicer wild-type and knockout ESCs correlated closely with independently derived, wild-type ESCs in the panel and clustered separately from differentiated cell types (Fig. 1C). In total, loss of miRNAs does not result in gross changes in preestablished cellular identity.

\section{Dicer knockout fibroblasts exhibit a let-7-regulated} oncofetal signature

Dicer knockout MSCs exhibit signatures for numerous miRNAs. From among these signatures, we focused on let-7 because it is an abundant tumor suppressor that represents the somatic counterpoint to the ESC-specific miR-290 family. In Dicer knockout MSCs, the top two statistically significant up-regulated genes were the oncofetal let-7 targets Igf2bp1 (up 48-fold by microarray) and Igf2bp2 (up 16-fold). By quantitative PCR (qPCR), we confirmed the up-regulation of these genes as well as Igf2bp3 and Hmga2, two additional oncofetal genes and targets of let-7 (Fig. 2A). The large magnitude of upregulation for all four genes was striking, ranging by qPCR from 11-fold for Hmga2 to 300-fold for Igf2bp2. Similarly, by Western blot, these genes were largely undetectable at the protein level in Dicer wild-type MSCs but became highly expressed in Dicer knockout MSCs (Fig. 2B). All four genes are let-7 targets, demonstrated by their down-regulation at the mRNA and protein levels by transfection with an siRNA duplex of let-7g (Fig. 2A,B). In contrast, neither Lin28a nor Lin28b was expressed in Dicer wild-type or knockout MSCs (Supplemental Table S2), suggesting that factors in addition to miRNA loss contribute to up-regulation of oncofetal genes.

Due to the magnitude of gene expression changes for these genes, we tested whether transcription contributes to this oncofetal signature. We carried out chromatin immunoprecipitation (ChIP) coupled with sequencing (ChIP-seq) for H3K4me3 and H3K36me3, associated with 
A

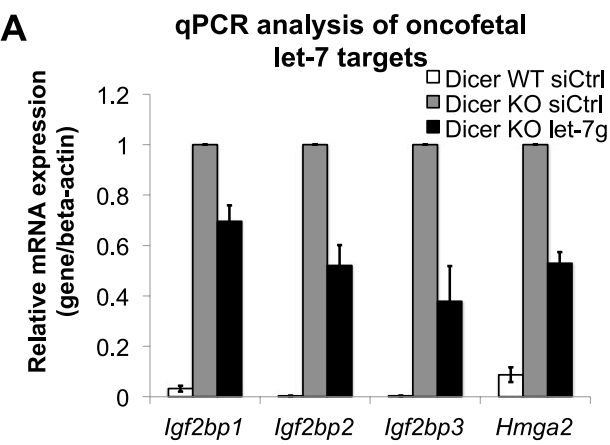

C

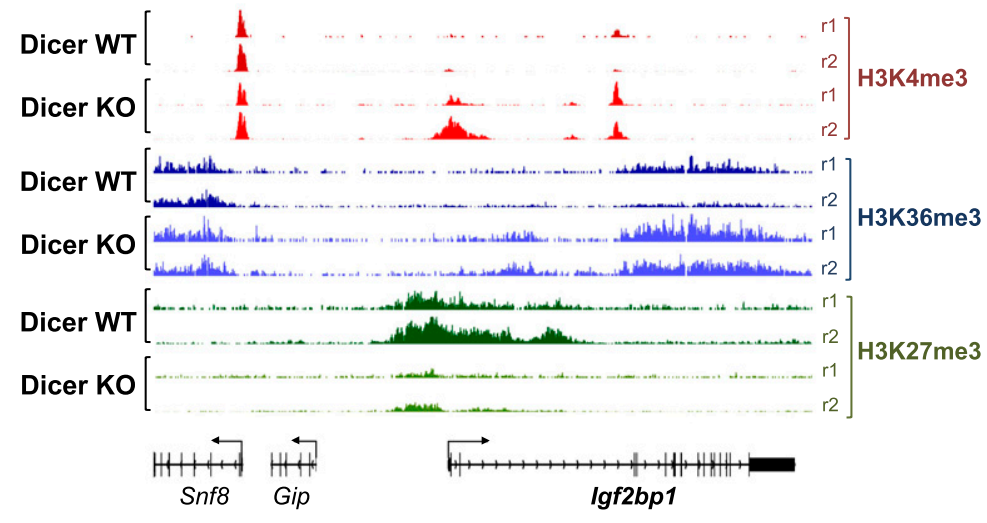

B

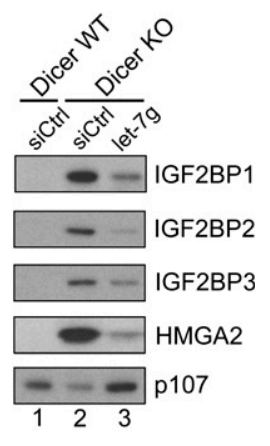

\section{S}

D
Figure 2. Dicer knockout (KO) MSCs up-regulate oncofetal genes. $(A, B)$ qPCR $(A)$ and Western blot analysis $(B)$ of oncofetal genes. p107 is shown as a loading control for Western blot. Error bars indicate standard error of the mean ( \pm SEM). (C) Normalized read counts for chromatin marks at Igf2bp 1 for Dicer wild-type (WT) and knockout MSCs. Two replicates ( $\mathrm{r} 1$ and $\mathrm{r} 2)$ are shown per sample. Within each chromatin mark (H3K4me3, H3K36me3, and H3K27me3), all samples are set to the same scale. Flanking genes are shown as controls. $(D)$ Box plot of $\log _{2}$ fold change in gene expression for all genes ("All"), all predicted let-7 targets ("Let-7"), genes enriched in H3K4me3 in Dicer knockout MSCs relative to Dicer wild-type MSCs ("H3K4me3 in Dicer $\left.\mathrm{KO}^{\prime \prime}\right)$, or overlapping genes from the latter two categories ("Let-7, H3K4me3 in Dicer KO"). P-values were calculated with a Wilcoxon rank-sum test. See also Supplemental Figure S2 and Supplemental Tables S2 and S3.

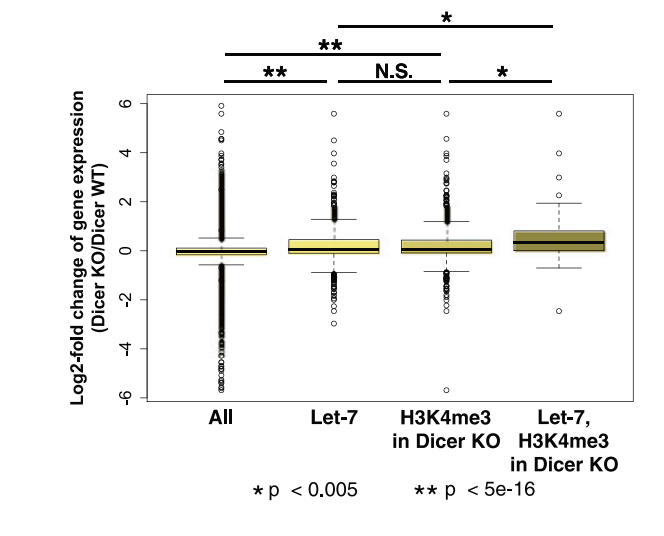

transcriptionally active genes, and $\mathrm{H} 3 \mathrm{~K} 27 \mathrm{me} 3$, associated with transcriptionally inactive genes (Supplemental Table S3). We confirmed that genes associated with H3K4me3 and H3K36me3 marks were expressed highly at the mRNA level relative to all genes, while genes associated with $\mathrm{H} 3 \mathrm{~K} 27 \mathrm{me} 3$ were expressed lowly relative to all genes (Supplemental Fig. S2A,B).

We inspected oncofetal genes for changes in these histone marks. For Igf2bp1 (Fig. 2C), Igf2bp2 (Supplemental Fig. S2E), and Igf2bp3 (Supplemental Fig. S2F), we observed broad peaks of H3K27me3 in Dicer wild-type MSCs, supporting the observation that these genes are "off" in miRNA-expressing cells. Conversely, in Dicer knockout MSCs, all three genes exhibited a loss of $\mathrm{H} 3 \mathrm{~K} 27 \mathrm{me} 3$ and concomitant gains in promoter-associated H3K4me3 and gene body-associated H3K36me3, demon- strating transcriptional activation of this family of genes. In comparison, the ChIP-seq density for four flanking genes (Tra2a, Tra2b, Snf8, and Gip) was indistinguishable between Dicer wild-type and knockout cells, consistent with the observation that these control genes are not differentially expressed upon Dicer loss (mean fold change $=$ 1.0, mean adjusted $P$-value $=0.88)$. Although Lin28a and Lin28b lost H3K27me3 density in Dicer knockout MSCs, they did not exhibit any detectable peaks of either H3K4me3 near their promoters or H3K36me3 in their gene bodies, suggesting that these two oncofetal genes are not transcriptionally activated, consistent with the absence of their expression by microarray.

A more global comparison of histone marks between genotypes also revealed Dicer-dependent changes in chromatin. Several thousand genotype-specific peaks for 
H3K4me3 and H3K36me3 were enriched in one genotype relative to the other (Supplemental Table S3). Specifically, 205 and 981 genes were marked with genotype-specific H3K4me3 peaks in Dicer wild-type and Dicer knockout MSCs, respectively, and generally exhibited higher expression in their respective genotypes (Supplemental Fig. S2C). Similarly, 357 and 147 genes were marked with genotype-specific H3K36me3 peaks in Dicer wild-type and Dicer knockout MSCs, respectively, and generally exhibited higher expression in their respective genotypes (Supplemental Fig. S2D).

To determine whether transcription contributes to upregulation of let-7 targets generally, we examined the expression of the overlap between predicted let-7 targets (813 genes) and genes enriched for H3K4me3 in Dicer knockout relative to Dicer wild-type MSCs (981 genes). These genes (63 genes) (Supplemental Table S7, "Let-7, H3K4me3 in Dicer $\mathrm{KO}^{\prime \prime}$ ), which include the Igf2bp1-3 family but not Hmga2, were more highly expressed than all predicted let-7 targets (Fig. 2D, cf. "Let-7" and "Let-7, H3K4me3 in Dicer KO") or all genes enriched for H3K4me3 in Dicer knockout relative to Dicer wild-type (Fig. 2D, cf. "H3K4me3 in Dicer KO" and "Let-7, H3K4me3 in Dicer KO"). In total, oncofetal let-7 targets are up-regulated, with transcriptional increases, in Dicer knockout MSCs.

\section{Add-back of let-7 to Dicer knockout fibroblasts identifies direct targets}

To expand our analysis of let-7 beyond known oncofetal targets and validate additional targets among computational predictions, we experimentally identified targets by reconstituting let-7 expression in Dicer knockout MSCs. To do so, we carried out mRNA sequencing (mRNA-seq) on polyA-selected total RNA isolated $48 \mathrm{~h}$ after transfection of Dicer wild-type MSCs with nontargeting control siRNA (siCtrl) or transfection of Dicer knockout MSCs with either siCtrl or synthetic let-7g siRNA duplex as a representative member of the let-7 family (Supplemental Table S4).

Comparison of Dicer wild-type and knockout MSCs by mRNA-seq recapitulated the exon microarray results and demonstrated specific gene expression changes with Dicer loss (Supplemental Fig. S3A). Relative to controltransfected Dicer wild-type MSCs, 1783 genes (FPKM [fragments per kilobase of exon per million fragments mapped] cutoff $\geq 0.1, q$-value $<0.05$ ) were up-regulated in control-transfected Dicer knockout MSCs with a median fold change of $\sim 2$, while 1746 genes (FPKM cutoff $\geq 0.1$, $q$-value $<0.05$ ) were down-regulated with a median fold change of $\sim 2$ (Supplemental Fig. S3A). miRNA targets were largely derepressed in Dicer knockout MSCs relative to Dicer wild-type MSCs (Supplemental Fig. S3B). More genes were differentially expressed by mRNA-seq than by exon microarray, probably due to the sensitivity of sequencing and the use of a single pair of Dicer wildtype and knockout clones in the mRNA-seq experiment, thus eliminating clonal heterogeneity.

Add-back of let-7 to Dicer knockout MSCs induced many changes in gene expression, including down-regulation of 1233 genes (FPKM cutoff $\geq 0.1, q$-value $<0.05$ ), with a median fold change of $\sim 2$, and the up-regulation of 1993 genes (FPKM cutoff $\geq 0.1, q$-value $<0.05$ ), with a median fold change of $\sim 2$ (Fig. 3A; Supplemental Fig. S3C). Let-7g-transfected cells clustered more closely with siCtrl-transfected Dicer knockout MSCs than with siCtrltransfected Dicer wild-type MSCs, indicating that changes in addition to let-7 loss, such as miR-15-loss or miR-199loss, contribute to the gene expression profile of Dicer loss (Supplemental Fig. S3D).

Analysis of expression changes of all predicted miRNA targets revealed a strong let-7 signature in let-7g-transfected Dicer knockout MSCs. The moving average of the TargetScan score for predicted let-7 targets increased significantly among genes that were most highly expressed in Dicer knockout siCtrl cells relative to siCtrl-transfected Dicer wild-type and let-7g-transfected Dicer knockout MSCs (Fig. 3A, side plot). In contrast, no signal was observed in this moving average for predicted targets of miR-15, indicating specificity of the let- $7 \mathrm{~g}$ siRNA. Global analysis of median gene expression changes in Dicer knockout MSCs transfected with let-7g revealed statistically significant signals for targets of let-7; miR-202, which shares a 6-mer seed with the let-7 family; and miR-196, whose seed sequence begins at position 2 of the let-7 seed sequence and thus is a seed-shifted relative of let-7 (Supplemental Fig. S3E). GSEA confirmed downregulation of let-7 targets upon transfection of let-7g siRNA (Supplemental Fig. S3F).

Add-back of let-7 should result in two types of changes: (1) direct repression of let-7 targets and (2) secondary effects downstream from let-7 targets, including genes induced by let- 7 add-back. To identify targets repressed directly by let-7, we overlapped genes that were upregulated following Dicer loss by mRNA-seq at $q$-value $<0.05$ (Fig. 3B, left panel, "Derepressed in Dicer KO"), down-regulated with add-back of let- $7 \mathrm{~g}$ at $q$-value $<0.05$ (Fig. 3B, left panel, "Repressed with let-7 add-back in Dicer $\mathrm{KO}^{\prime \prime}$ ), and predicted by TargetScan to be let-7 targets (Fig. 3B, left panel, "Predicted targets of let-7"). This triple overlap identified 122 genes (listed in Supplemental Table S7) that included Hmga2 and the Igf2bp1-3 family discussed above, an enrichment of 28 -fold above background. We randomly selected seven genes from this set for validation by qPCR and, for all seven genes, confirmed up-regulation upon Dicer loss and down-regulation following transfection of let-7g (Fig. 3C). However, repression of predicted targets by add-back of let-7 was only partially reversible and may reflect either transcriptional effects, a requirement for cotargeting by additional seed families, or transfection of synthetic let-7 at a concentration below endogenous levels. Hereafter, we refer to this set of 122 genes as "high-confidence" targets of let-7.

To test whether up-regulation of "high-confidence" targets could be generalized to a second cell type, we analyzed microarray gene expression data (Supplemental Table S5) from sarcoma cell lines that are $\mathrm{Kras}^{\text {G12D }}$; $\operatorname{Trp} 53^{-/-}$; Dicer $1^{f-}$ (heterozygous) and in which let-7 is the most abundant seed family or Kras ${ }^{G 12 D} ; \operatorname{Tr} p 53^{-/-} ;$Dicer $1^{-/-}$ (knockout) (Ravi et al. 2012). Indeed, "high-confidence" 
Gurtan et al.

A

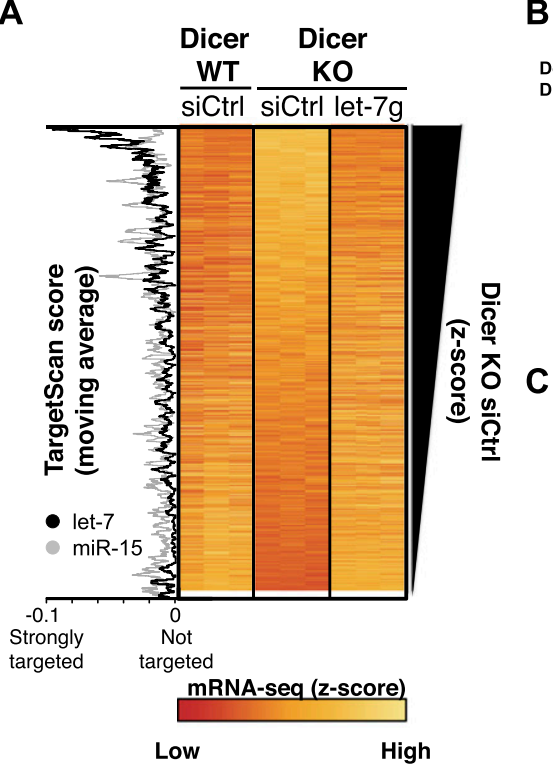

B
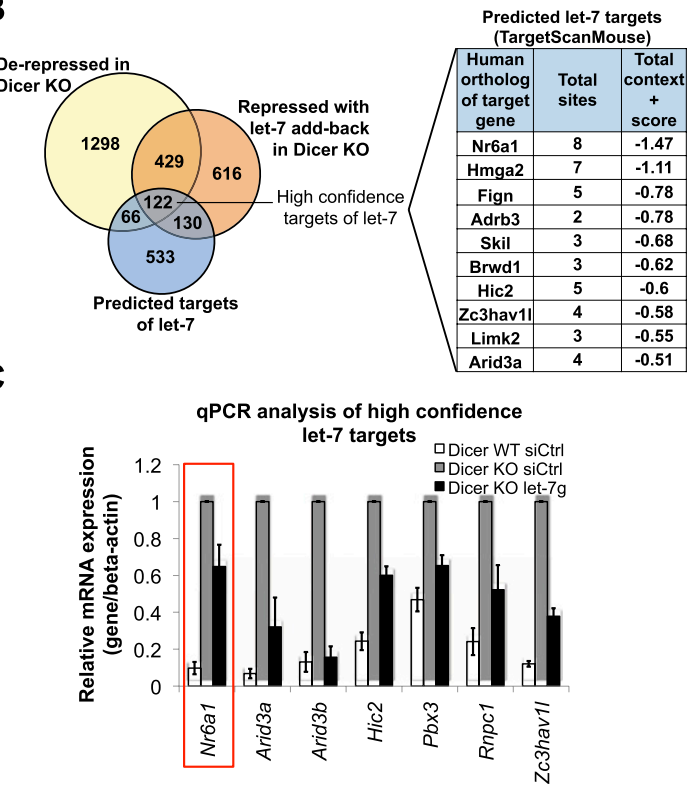

D

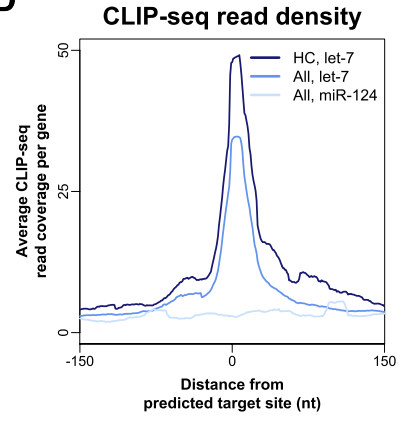

$\mathbf{E}$

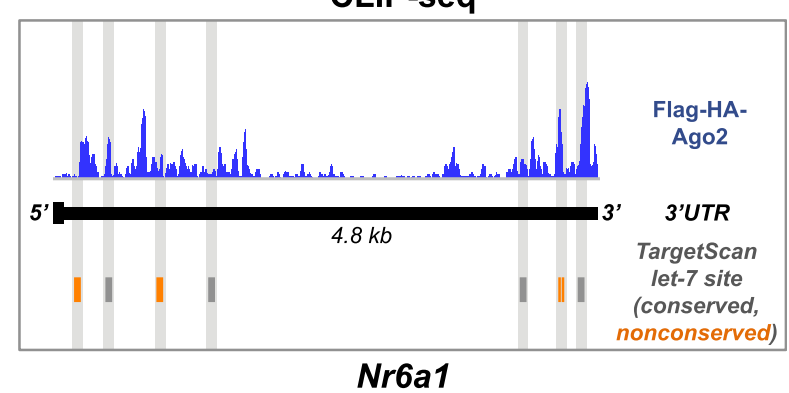

Figure 3. Identification of let-7 targets by let-7 add-back to Dicer knockout (KO) MSCs. (A) Heat map of expression Z-scores in transfected Dicer wild-type (WT) and knockout MSCs for all genes expressed at FPKM $\geq 0.1$. Genes are ranked from highest (top) to lowest (bottom) Z-score in Dicer knockout MSCs. A 10-gene moving average of TargetScan scores is shown in the left plot for let-7 (black) and miR-15 (gray). (B) Overlap of genes up-regulated at $q$-value $<0.05$ in siCtrl-transfected Dicer knockout MSCs relative to siCtrl-transfected Dicer wild-type MSCs ("Derepressed in Dicer KO"), down-regulated at $q$-value $<0.05$ in let-7g-transfected Dicer knockout MSCs relative to siCtrl-transfected Dicer knockout MSCs ("Repressed with let-7 add-back in Dicer KO"), and predicted by TargetScan to be conserved targets of let-7 ("Predicted targets of let-7"). Top predicted let-7 targets in TargetScanMouse are shown in the table on the right. (C) qPCR analysis of high-confidence targets of let-7 identified in triple overlap above. Error bars indicate the SEM. (D) Metaplot of CLIP-seq read density. CLIP-seq reads were aligned to TargetScan-predicted sites. The average CLIP-seq read coverage per gene was plotted relative to let-7 target sites within the 3' UTRs of high-confidence targets of let-7 ("HC, let-7"), let-7 target sites within the 3' UTRs of all TargetScanpredicted targets of let-7 ("All, let-7"), or miR-124 target sites within the 3' UTRs of all TargetScan-predicted targets of let-7 ("All, miR$\left.124^{\prime \prime}\right)$. The analysis was carried out for genes with FPKM $\geq 0.1$ in either Dicer wild-type or knockout MSCs transfected with siCtrl. (E) CLIP-seq density along the 3' UTR of Nr6a1. The blue histogram indicates CLIP-seq density; gray tick marks indicate the location of TargetScan-predicted, conserved let-7 sites; and orange tick marks indicate the location of TargetScan-predicted, nonconserved let-7 sites. The length of the 3' UTR is indicated. See also Supplemental Figures S3 and S4 and Supplemental Tables S4 and S6.

targets of let-7 were largely up-regulated with Dicer loss in sarcoma cells relative to control genes (Supplemental Fig. S3G). The oncofetal genes Igf2bp1 and $I g f 2 b p 3$ were strongly up-regulated, as were many of the genes that we validated in MSCs by qPCR (Supplemental Fig. S3H, note the logarithmic Y-axis). Upregulation of Hmga2 and Igf2bp2 was not observed in sarcoma cells, likely due to already high basal expression in muscle (Li et al. 2012), the tissue type from which these cells were derived.

Thus far, we defined "high-confidence" targets of let-7 based on gene expression. To determine whether these genes are directly bound by the RNA-induced silencing complex (RISC) loaded with let-7, we carried out crosslinked immunoprecipitation (CLIP) sequencing (CLIPseq) (Chi et al. 2009). To do so, we infected Dicer wild-type MSCs with lentivirus encoding doxycycline-inducible FlagHA-Ago2 or untagged Ago2 as a negative control, selected transduced cells with hygromycin, and induced transgene expression with doxycycline. We then sequenced UV-cross-linked RNA that was isolated from Flag-HAAgo2- and untagged Ago2-expressing cells by sequential anti-Flag and anti-HA immunoprecipitation (Supplemental Table S6). 
In Flag-HA-Ago2-expressing cells, a metagene plot at TargetScan-predicted, conserved let-7 target sites demonstrated higher CLIP-seq signal per gene for "highconfidence" let-7 targets compared with all conserved TargetScan let-7 targets expressed $\geq 0.1$ FPKM (Wilcoxon rank-sum test $P$-value $\left.=1.4 \times 10^{-22}\right)($ Fig. 3D). In contrast, no peak in signal was observed at target sites of miR-124, a miRNA not expressed in MSCs, within TargetScan-predicted let-7 targets (Fig. 3D) or at let-7 sites in CLIP-seq carried out from cells expressing untagged Ago2 (data not shown). Inspection of individual 3' UTRs demonstrated CLIP-seq peaks at both conserved and nonconserved TargetScan-predicted let-7 sites for Nr6a1 (Fig. 3E), a gene characterized in further detail below. We also observed peaks at let-7 target sites within the 3' UTRs of Igf2bp 1 (Supplemental Fig. S4A), Arid3a (Supplemental Fig. S4B), $\mathrm{Pb} \times 3$ (Supplemental Fig. S4C), and numerous other genes, including those validated in Figure 3C.

Approximately $78 \%$ of "high-confidence" targets of let-7 exhibited five or more reads within $5 \mathrm{nt}$ of their predicted let-7 target sites. For all TargetScan targets, $\sim 63 \%$ of genes exhibited five or more reads at predicted let-7 target sites. In contrast, for both "high-confidence" let-7 targets and all predicted let- 7 targets, only $\sim 30 \%$ of genes possessing target sites for miR-124 exhibited five or more reads at predicted miR-124 sites. CLIP-seq from Dicer wild-type MSCs likely underestimates binding of the RISC to those genes that are not transcribed or are very lowly expressed. For example, Igf2bp2, validated previously (Alajez et al. 2012), and Igf2bp3 are more strongly repressed than Igf2bp1 upon let-7 add-back but are below the five-read cutoff by CLIP-seq in Dicer wild-type MSCs. For multiple genes, CLIP-seq peaks were also observed at predicted sites for miRNAs other than let-7, indicating regulation by multiple miRNA seed families. In total, we experimentally identified numerous let-7 targets functionally by let-7 add-back to Dicer knockout MSCs and independently confirmed that these genes are strongly enriched for Ago2-binding at let-7 sites.

\section{Let-7 targets comprise a mid-gestation embryonic program}

Since miRNAs may regulate networks of functionally related genes, we examined whether "high-confidence" targets of let-7 possess any common characteristics. Many of these genes, such as Igf2bp1-3, Hmga2, Pbx3, and Arid3b, are known to be expressed in the embryo (Hirning-Folz et al. 1998; Hansen et al. 2004; Takebe et al. 2006; Vitobello et al. 2011), in line with the hypothesis that mammalian let-7 regulates development. We also identified numerous oncogenes, including Plagl2, which is overexpressed in glioma and colorectal cancer (Zheng et al. 2010), and Arid3a, which drives bypass of RASinduced senescence (Peeper et al. 2002). Furthermore, several "high-confidence" let-7 targets, including but not limited to Plag12, Arid3a, and oncofetal genes, are known to peak in expression around mid-gestation (approximately embryonic day 8.5 [E8.5] to E10.5). Importantly, this timing distinguishes these genes from Oct4 and other pluripotency markers that are expressed in ESCs, down-regulated upon differentiation, and inactive in Dicer knockout MSCs.

To systematically determine whether "high-confidence" targets of let-7 exhibit a mid-gestation embryonic signature, we profiled these genes in a published time course of gene expression in whole mouse embryos (Irie and Kuratani 2011). We restricted our analysis to genes that were expressed in at least one of the time points in the data set. The majority of "high-confidence" targets of let-7, including Hmga2 and the Igf2bp1-3 family, are expressed mid-gestation and steadily decrease as embryogenesis progresses (Fig. 4A). To quantify these changes, we plotted the mean $Z$-score for each gene (the expression of each gene at each time point normalized to the mean expression and standard deviation of the gene across all time points). These genes peak in expression at E8.5-E10.5 and subsequently decline (Fig. 4B), thus anti-correlating with the expression of let-7, which becomes detectable by Northern blot in whole mouse embryos at E10.5, plateaus by E14.5, and remains high postnatally (Fig. 4B-E, dashed line; Schulman et al. 2005). In contrast, the set of all conserved let-7 targets predicted by TargetScan and expressed in at least one time point in the whole mouse embryo data set was largely indistinguishable from background (Fig. 4C). We confirmed the anti-correlation between let- 7 and its "high-confidence" targets in a second, independent published time course of mouse embryonic limb bud development (Taher et al. 2011). As before, the majority of "highconfidence" targets decreased as the embryonic limb bud developed (Supplemental Fig. S5A). On average, these genes anti-correlated with let-7 (Supplemental Fig. S5B), while, in contrast, the set of all conserved let-7 targets predicted by TargetScan was largely indistinguishable from background (Supplemental Fig. S5C). In total, members of the "highconfidence" let-7 target set peak in expression around E8.5 and anti-correlate with let-7 during development.

Add-back of let-7 in Dicer knockout MSCs also induces the expression of many genes, which are likely "indirect targets" downstream from genes repressed directly by let-7. In the whole mouse embryo, we tested whether "indirect targets" of let-7 exhibited a specific pattern. "Indirect targets" were induced as the embryo matured, correlating positively with let-7 and anti-correlating with "highconfidence" targets of let-7 (Fig. 4D). This positive correlation was also observed in the mouse embryonic limb bud (Supplemental Fig. S5D). In total, let-7 controls a mid-gestation embryonic program in somatic cells by directly repressing genes that peak mid-gestation and indirectly inducing genes that become activated as the embryo matures.

Nr6a1, an embryonic transcriptional repressor, is a target of let-7 and represses gene expression in Dicer knockout MSCs

The gene Nr6a1, also known as Germ cell nuclear factor $(G C N F)$, was notable among "high-confidence" let-7 targets, since it meets the criteria of a classical developmental target of let-7. Nr6a1 is an embryonically expressed 
A

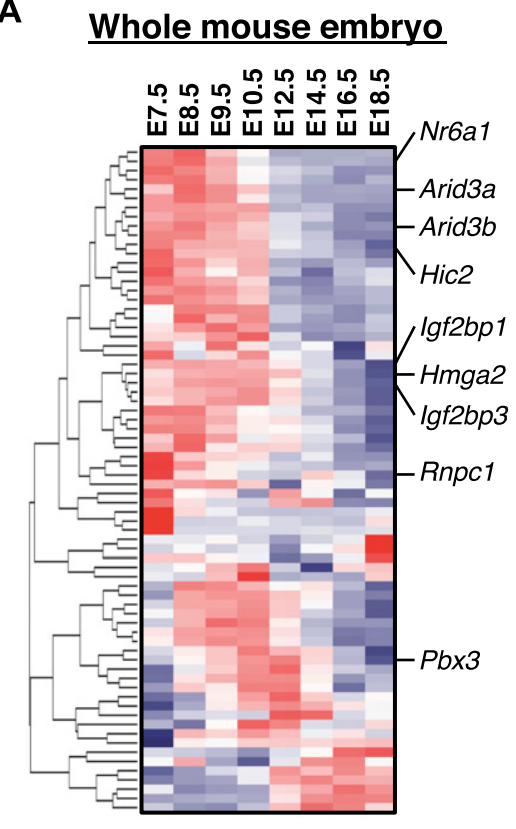

B

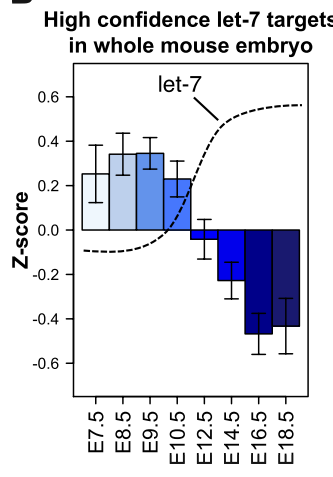

D

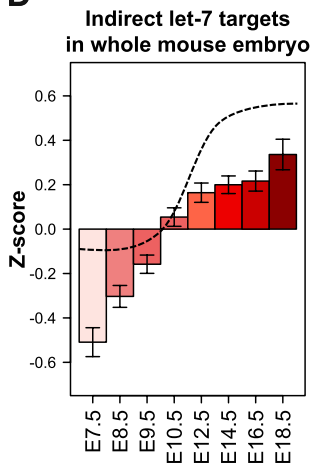

C

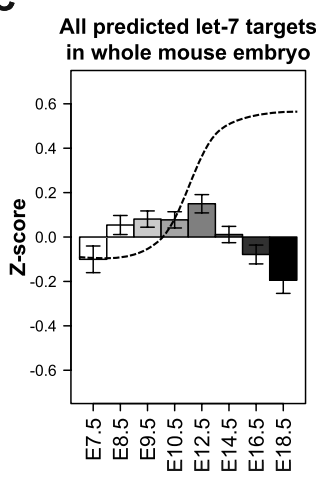

$\mathbf{E}$

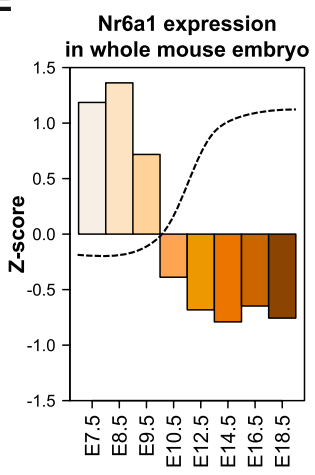

Figure 4. Let-7 targets are dynamically expressed in the whole mouse embryo. (A) Heat map of individual high-confidence let-7 targets in the whole mouse embryo from E7.5 to E18.5 (Irie and Kuratani 2011). Genes validated by qPCR in this study are labeled on the right. $(B-E)$ Average expression $Z$-score for high-confidence let-7 targets $(B)$, all predicted conserved TargetScan targets of let-7 $(C)$, indirect targets of let-7 (D), and Nr6a1 (E) in the whole mouse embryo from E7.5-E18.5 (Irie and Kuratani 2011). The black dashed line shows let-7 expression. Error bars indicate the SEM. See also Supplemental Figure S5.

sequence-specific transcriptional repressor and orphan nuclear receptor (Fuhrmann et al. 2001). Like many targets of let-7, such as the nematode nuclear steroid receptor daf12 (Hammell et al. 2009), Nr6a1 is critical to development, during which it inhibits the pluripotency factor Oct4 $(\mathrm{Gu}$ et al. 2005). Germline deletion of Nr6a1 results in embryonic lethality mid-gestation due to failures in neural tube closure and somitogenesis (Chung et al. 2001). Nr6a1 is the top-predicted target of let-7 by TargetScan and possesses eight let-7 seed matches in its 3' UTR (Fig. 3B, right panel). By qPCR, we confirmed up-regulation of Nr6a1 with miRNA loss and its down-regulation following add-back of let-7 (Fig. 3C, boxed in red). Similarly, Nr6a1 is upregulated 10-fold in Dicer knockout sarcoma cells (Supplemental Fig. $\mathrm{S} 3 \mathrm{H}$ ). We also demonstrated its interaction with the RISC at predicted let-7 target sites (Fig. 3E), indicating that Nr6a1 is a direct target of let-7. Finally, Nr6a1 is part of the mid-gestation signature regulated by let-7, with a peak in expression around E8.5 and a subsequent decrease anti-correlating with let-7 in the whole mouse embryo (Fig. 4E) and in the mouse embryonic limb bud (Supplemental Fig. S5E).

Having identified the transcriptional repressor Nr6a1 as part of a mid-gestation program regulated by let- 7 in adult fibroblasts, we combined knockdown and overexpression experiments to determine whether Nr6a1 in turn regulates gene expression in Dicer knockout MSCs
(Fig. 5A; Supplemental Table S4). We transfected Dicer knockout MSCs, which are "NR6A1-high," with siRNA against Nr6a1 (Supplemental Fig. S6A) and profiled gene expression by mRNA-seq (Supplemental Fig. S6B). With a knockdown efficiency of $\sim 75 \%$, we observed differential expression of 1601 genes (FPKM cutoff $\geq 0.1, q$-value $<$ 0.05). Of these, 978 were up-regulated with a median fold change of 1.8 (Supplemental Fig. S6B). The observation that depletion of NR6A1 results in majority up-regulation of genes suggests that NR6A1 acts as a transcriptional repressor in Dicer knockout MSCs.

Next, to identify genes directly regulated by NR6A1 and uncouple it from accompanying gene expression changes observed with Dicer loss, we overexpressed Flag-HANR6A1 in Dicer wild-type MSCs (Supplemental Fig. S6C), which are "NR6A1-low." By mRNA-seq, we observed differential expression of 104 genes (FPKM cutoff $\geq 0.1$, $q$-value $<0.05$ ), of which a majority were down-regulated with a median change of 5.5-fold (Supplemental Fig. S6D), consistent with a transcriptionally repressive activity for NR6A1. Next, we characterized the expression of these Flag-HA-NR6A1-responsive genes in Dicer knockout MSCs. To do so, we restricted our analysis to genes differentially expressed at a $q$-value $<0.05$ within each data set and expressed at an FPKM $\geq 0.1$ across all three data sets (leading to a total of 76 genes responsive to expression of Flag-HA-NR6A1, 1556 genes responsive to knockdown of 
A

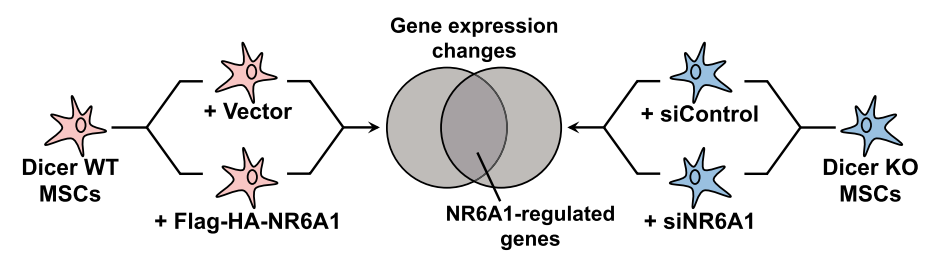

B

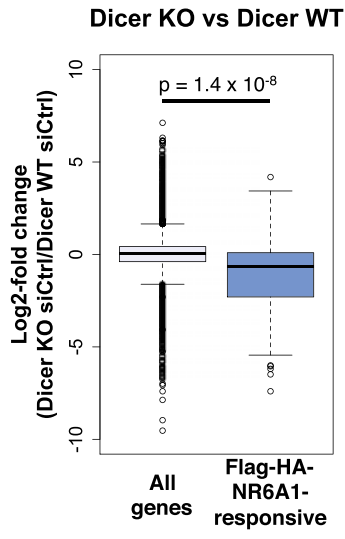

D

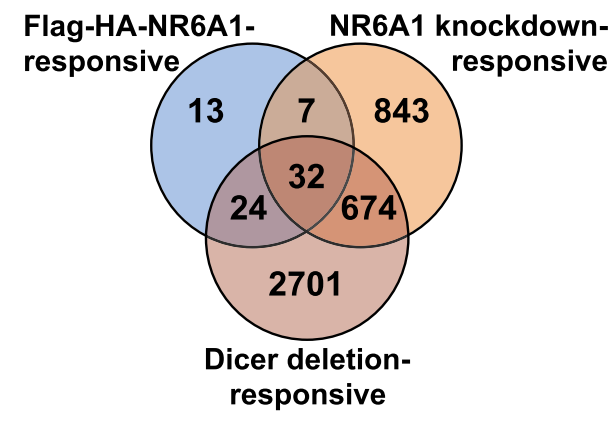

C
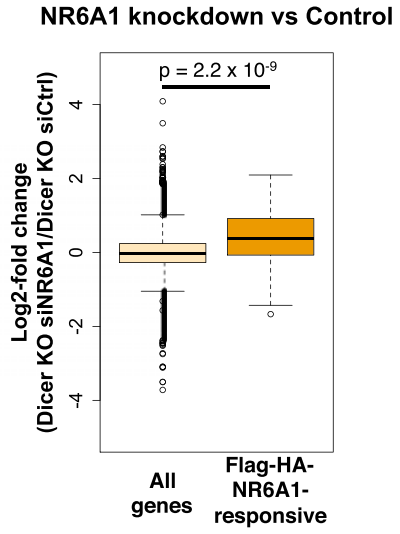

E

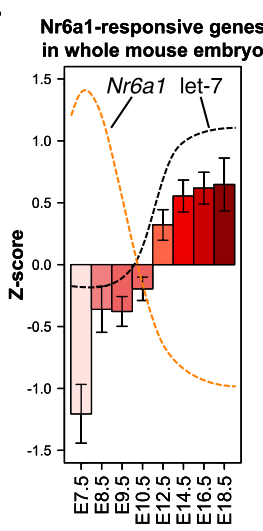

Figure 5. Gain-of-function and loss-of-function approaches identify genes regulated by let-7 target NR6A1. (A) Schematic of experimental design. (From left) Dicer wild-type (WT) MSCs ("NR6A1low") were infected with pMMP-puro retrovirus encoding vector control or Flag-HA-NR6A1, selected with puromycin, and passaged for several weeks prior to isolation of total RNA for polyAselected mRNA-seq. (From right) Dicer knockout (KO) MSCs ("NR6A1-high") were transfected with control nontargeting siRNA (siCtrl) or siRNA against Nr6a1 (siNR6A1) $48 \mathrm{~h}$ prior to isolation of total RNA for polyA-selected mRNA-seq. (B) Box plot of gene expression changes in siCtrl-transfected Dicer knockout versus siCtrl-transfected Dicer wild-type MSCs for all genes ("All genes") or genes differentially expressed upon overexpression of Flag-HA-NR6A1 ("Flag-HA-NR6A1-responsive"). An FPKM cutoff $\geq 0.1$ was used for all compared data sets. $P$-value was calculated by Wilcoxon rank-sum test. $(C)$ Box plot of gene expression changes in siNR6A1-transfected Dicer knockout versus siCtrl-transfected Dicer knockout MSCs for all genes ("All genes") or genes differentially expressed upon overexpression of Flag-HA-NR6A1 ("Flag-HA-NR6A1-responsive"). An FPKM cutoff $\geq 0.1$ was used for all compared data sets. $P$-value was calculated by Wilcoxon rank-sum test. $(D)$ Triple overlap of Flag-HA-NR6A1-responsive, NR6A1 knockdown-responsive, and Dicer deletionresponsive gene sets. $(E)$ Average $Z$-score in the whole mouse embryo time course for 32 NR6A1-responsive genes defined in $D$. The orange dashed line shows Nrba1 expression, and the black dashed line shows let-7 expression. Error bars indicate the SEM. See also Supplemental Figure S6 and Supplemental Table S4.
Nr6a1, and 3431 genes responsive to Dicer loss). Flag-HANR6A1-responsive genes, listed in Supplemental Table S7, were largely repressed in Dicer knockout MSCs (Fig. 5B) and subsequently induced in Dicer knockout MSCs following knockdown of Nr6a1 (Fig. 5C), demonstrating that Nr6a1 is responsible for their down-regulation with miRNA loss. Thirty-two genes, defined hereafter as "NR6A1-responsive," were common in the overlap of changes observed upon overexpression of Flag-HA-NR6A1, loss of Dicer, and knockdown of endogenous Nr6a1 (Fig. 5D; Supplemental Table S7), representing an enrichment of 12 -fold over background. Of these 32 genes, 13 were also responsive to let-7 (Supplemental Table S7). This partial overlap suggests that the activity of Nr6a1 is not restricted to targets of let-7. Notably, given that Nr6a1 is a transcriptional repressor, it is unlikely to account for the transcriptional induction of let- 7 targets, such as Igf2bp1, observed in Dicer knockout MSCs. Furthermore, overexpression of Nr6a1 alone in Dicer wild-type MSCs, which are "Nr6a1-low," results in the differential expres- sion of $\sim 100$ genes, in contrast to the $>1000$ transcriptional changes that we observed with Dicer loss. These observations suggest that other miRNA-regulated factors in addition to or in place of Nr6a1 contribute to changes in histone marks.

Since Nr6a1 is normally expressed during development, we examined the embryonic expression of NR6A1responsive genes. These genes correlated inversely with Nr6a1, with low expression mid-gestation that increased as the whole mouse embryo (Fig. 5E) and embryonic limb bud (Supplemental Fig. S6E) matured. In total, we identified Nr6a1 as a let-7 target that mediates secondary transcriptional gene expression changes in Dicer knockout MSCs.

\section{Genome-wide binding profile identifies direct targets} of NR6A1

Having identified genes functionally responsive to Nr6a1, we next carried out ChIP-seq of Flag-HA-NR6A1 to 
identify genes that are directly bound (Supplemental Fig. S7A; Supplemental Tables S3, S7). We identified 9223 enriched regions, corresponding to a total of 5210 bound or nearby genes (Fig. 6A). Approximately $19 \%$ of peaks overlapped with promoters, defined as regions spanning 5 $\mathrm{kb}$ upstream of to $1 \mathrm{~kb}$ downstream from annotated transcription start sites (TSSs); $38 \%$ of peaks were in gene bodies; and $43 \%$ of peaks were intergenic (Fig. 6A,C). Peaks in all three categories were strongly enriched for the sequence CAAG(G/T)TCA (Fig. 6B), reported previously to be part of the consensus recognized by NR6A1 (Yan et al. 1997). We also identified additional enriched sequences (Supplemental Fig. S7B) that may be recognized by interacting partners of NR6A1. Interestingly, Flag-HA-NR6A1 was bound to three oncofetal genes (Hmga2, Igf2bp2, and Igf2bp3), suggesting crossregulation of genes within mid-gestation programs. However, the mRNA levels of these oncofetal genes did not change upon induction of Flag-HA-NR6A1 or knockdown of Nr6a1, suggesting the presence of additional regulators responsible for their differential expression.

Of the 32 NR6A1-responsive genes that were repressed in Dicer knockout cells and derepressed following knockdown of NR6A1, 26 were bound by NR6A1 (Supplemental Table S7), representing an enrichment of 2.6-fold over background $\left(\chi^{2}\right.$ test, $P$-value $\left.=6.0 \times 10^{-7}\right)($ Supplemental Fig. S7C), and nine of these genes possessed the NR6A1 consensus motif in either the promoter, gene body, or distal intergenic region. Among the 32 NR6A1-responsive genes (Fig. 6D, "Responsive"), those that were bound by NR6A1 (Fig. 6D, "Responsive, bound") were more strongly repressed upon overexpression of Flag-HANR6A1, particularly if the binding site possessed the NR6A1 consensus motif (Fig. 6D, "Responsive, bound, motif"). Genes bound by NR6A1, especially in regions containing the consensus site, were more strongly derepressed upon knockdown of NR6A1 in Dicer knockout MSCs (Fig. 6E). Finally, NR6A1-bound genes that were repressed upon overexpression of Flag-HA-NR6A1 anticorrelated with NR6A1 in the whole mouse embryo (Supplemental Fig. S7D) and in the mouse embryonic limb bud (Supplemental Fig. S7E). In total, these results identify direct targets of NR6A1 in MSCs and demonstrate the anti-correlation of these targets with NR6A1 expression during embryonic development.

\section{Discussion}

We characterized gene expression in immortalized bone marrow-derived Dicer-deficient somatic fibroblasts and
A

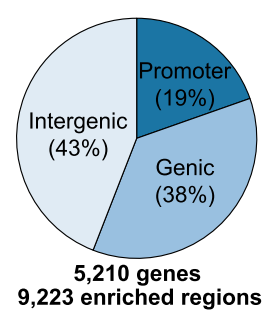

B

\begin{tabular}{|l|c|c|c|}
\hline Category & \multicolumn{1}{|c|}{ Motifi } & E-value & $\begin{array}{c}\text { Rank } \\
\text { in category }\end{array}$ \\
\hline Promoter & CAAGGTCA & $3.1 \mathrm{e}-63$ & 1 \\
\hline Genic & CAAGTTCA & $8.1 \mathrm{e}-198$ & 2 \\
\hline Intergenic & CAAGTCA & $1.8 \mathrm{e}-238$ & 3 \\
\hline Published & TCAAGTCA & - & - \\
\hline
\end{tabular}

C

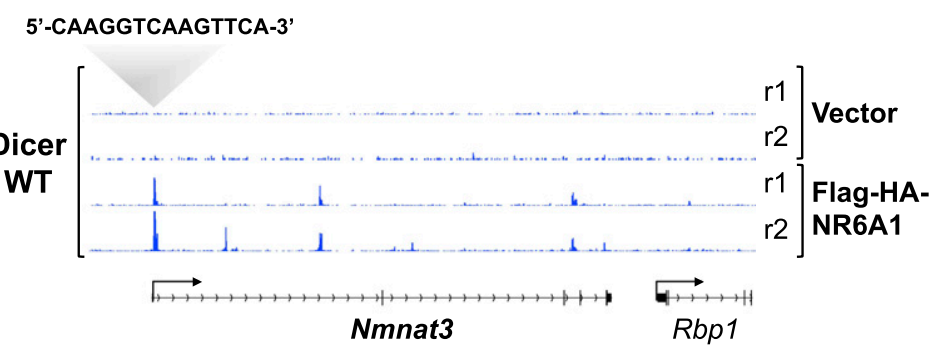

D

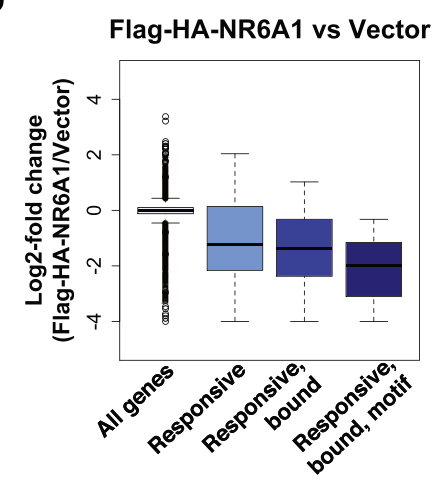

E

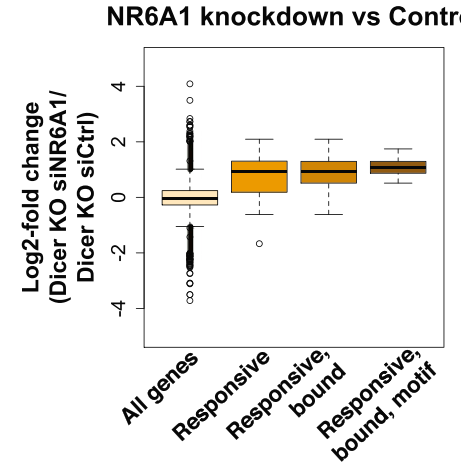

Figure 6. Global genomic profile of NR6A1 binding. (A) Summary of binding data for Flag-HANR6A1. In total, 9223 sites are bound genome-wide and map to either promoter-proximal $(5 \mathrm{~kb}$ upstream of to $1 \mathrm{~kb}$ downstream from the TSS), genic (excluding promoter-proximal regions), or intergenic regions. Associated genes were defined as those genes proximal to promoter peaks, overlapping with genic peaks, or closest to intergenic peaks. In total, 5210 genes are associated with or near Flag-HA-NR6A1-binding sites. The called regions represent the intersection of two clonal replicates. $(B)$ Motifs identified by DREME analysis in each category (promoter, genic, and intergenic) summarized in $A$. The motifs are ranked by enrichment score ( $E$-value) within each category (rank in category). The published motif is also shown (Yan et al. 1997). (C) Normalized read counts of two clonal replicates ( $\mathrm{r} 1$ and $\mathrm{r} 2$ ) of vector control or FlagHA-NR6A1 at Nmnat3, a gene responsive to FlagHA-NR6A1 overexpression, NR6A1 knockdown, and Dicer loss. The promoter-proximal site includes two tandem NR6A1 consensus motifs (shown above the read counts). A flanking gene is shown as a control. $(D, E)$ Box plots of gene expression changes in FlagHA-NR6A1-overexpressing Dicer wild-type (WT) MSCs versus vector-only Dicer wild-type MSCs (D) or siNR6A1-transfected Dicer knockout (KO) versus siCtrl-transfected Dicer knockout MSCs $(E)$ for all genes ("All genes"), the 32 genes responsive to NR6A1 as defined by triple overlap ("Responsive"), genes responsive to and bound by NR6Al ("Responsive, bound"), or the subset of NR6A1 consensus motifcontaining genes responsive to and bound by NR6A1 ("Responsive, bound, motif"). See also Supplemental Figure S7 and Supplemental Tables S3 and S4. 
observed large-magnitude changes in gene expression following loss of miRNAs. A subset of these effects was reinforced transcriptionally, notably for three archetypal oncofetal genes (Igf2bp1-3), indicating that the large fold changes observed in Dicer knockout MSCs for this family are attributable to not only disruption of miRNA-mRNA interactions (often responsible for only modest effects), but also transcriptional feedback that amplifies expression of let- 7 targets. Hence, microscale "fine-tuning" activity at the level of miRNA-mRNA interactions masks, in aggregate, macro-level transcriptional effects on gene expression.

Dicer knockout MSCs retain their mesenchymal identity, indicating that miRNAs do not primarily govern preestablished cell identity. This finding, in conjunction with the requirement for miRNAs during active differentiation and development, suggests a role for miRNAs primarily in the transition between cell states. Consistent with this possibility, miRNAs modulate the dynamics of gene expression to regulate cellular transitions and physiological robustness in numerous model systems (Herranz and Cohen 2010; Ebert and Sharp 2012). If the nearly universally reported stress sensitivity of miRNAdeficient cells is reframed as a general requirement for miRNAs in stimulus response, regardless of the nature of the stimulus, then the observation that miRNA loss results in embryonic lethality is consistent with the notion that miRNA-deficient cells cannot respond properly to developmental stimuli.

In the context of the immortalized cell lines reported here, let-7 represses an embryonic program distinct from pluripotency and related instead to a mid-gestation network that may regulate proliferative and metabolic pathways. In addition to known oncofetal let-7 targets, this program includes oncogenes that have not previously been associated with embryonic development or let-7, such as Plag12 (Zheng et al. 2010) and Arid3a (Peeper et al. 2002). Both genes peak mid-gestation in the whole mouse embryo. Thus, these genes may further populate the let7-regulated oncofetal network.

The up-regulation of a mid-gestation program in miRNAdeficient somatic cells suggests that the inactivation of these embryonic genes in adult tissue is maintained in a deliberately reversible state. Several of these genes, such as Hmga2 and Igf2bp1, play important roles in metabolism and proliferation (Viswanathan et al. 2009; Viswanathan and Daley 2010; Zhu et al. 2010; Frost and Olson 2011) and may be transiently activated, perhaps through down-regulation of let-7 or other miRNAs in somatic tissue, to promote injury repair or growth. This possibility is supported by a recent study demonstrating the importance of an HMGA2-IGF2BP2 axis in muscle regeneration in adult animals (Li et al. 2012). The overexpression of these oncofetal genes in tumors may reflect the inappropriate ectopic activation of an otherwise native, context-specific process regulated dynamically by miRNAs. Notably, the up-regulation of this discrete, embryonic program is compatible with mesenchymal cell identity, consistent with previous reports that gene expression consists of separable modules that can be coactivated in various permutations (Wong et al. 2008; Kim et al. 2010).

Our results also extend the current model of let-7 function in mammalian development. Our data suggest a stage in the embryo, from E8.5 to E10.5, in which the embryo has progressed well past a naive ESC state, thus shutting off the miR-290 family, but has not yet globally activated let-7 (Fig. 7). This possibility is consistent with recent findings that let-7 promotes development of the emerging mesoderm and ectoderm of mouse and Xenopus embryos (Colas et al. 2012). We postulate that let-7 plays a role beyond inhibition of pluripotency and represses mid-gestation programs to ensure a forward momentum during development, consistent with observations in nematodes that let-7 and lin- 4 mutant animals reiterate post-pluripotency larval stages (Lee et al. 1993; Reinhart et al. 2000).

Related to the role of let-7 in developmental timing, we identified Nr6a1, a potential functional ortholog of nematode daf-12, as a target of let-7. In our study, Nr6a1 represses genes that become activated as the embryo matures, suggesting that precise dosage of Nr6a1 is critical to developmental timing. Consistent with this observation, overexpression of Nr6a1 results in posterior defects and altered somite formation in Xenopus embryos (David et al. 1998). Thus, Nr6a1 and let-7 are likely sequentially activated and mutually antagonistic to ensure the proper chronology of gene expression in the midgestation embryo.

In conclusion, our findings support the hypothesis that the tumor-suppressive properties of let-7 are coupled to its repression, in somatic tissues, of metabolic embryonic programs. Furthermore, the global loss of miRNAs in adult tissue leads to transcriptional effects that reinforce specific gene expression programs. From a therapeutic standpoint, the inhibition of tumor growth may not be feasible by the inactivation of only a single miRNA target and may instead require delivery of individual synthetic tumor-suppressive miRNAs to inactivate full gene expression networks.

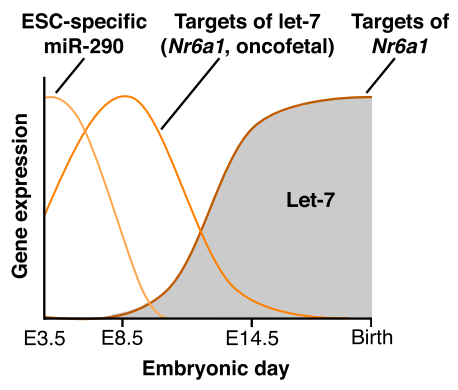

Figure 7. Summary of let-7 and target expression in the whole mouse embryo. Let-7 targets peak mid-gestation (around E8.5E10.5), after down-regulation of the ESC-specific miR-290 family. Expression of mature let-7 becomes detectable in the whole embryo around E10.5 and steadily increases in level, concomitant with down-regulation of high-confidence let-7 targets such as Nr6a1, identified in this study. Targets of Nr6a1 identified in this study increase as the embryo matures and positively correlate with let-7 in the whole mouse embryo. 


\section{Materials and methods}

Complete protocols are provided in the Supplemental Material and are also available on request. Microarray and sequencing data are available under Gene Expression Omnibus accession number GSE44163.

\section{Cell culture conditions}

Murine MSCs were passaged in $\alpha$-MEM supplemented with $10 \%$ FBS and penicillin/streptomycin as described previously (Gurtan et al. 2012; Ravi et al. 2012). Sarcoma cells were passaged in DMEM supplemented with 10\% FBS, L-glutamine, and penicillin/streptomycin as described previously (Ravi et al. 2012).

\section{Transfections and infections}

For siRNA transfections, cells were transfected with HiPerfect reagent (Qiagen) and $20 \mathrm{nM}$ siCtrl (Non-Targeting siRNA No. 2, Thermo Scientific Dharmacon), synthetic let-7g siRNA synthesized as perfectly complementary siRNA duplex (custom RNA synthesis service, Thermo Scientific Dharmacon), or gene-specific siRNA against Nr6a1 (Qiagen). Forty-eight hours after transfection, cells were harvested. Total RNA was isolated with an RNeasy kit (Qiagen) for qPCR or mRNA-seq. Total protein was isolated with radio immunoprecipitation assay (RIPA) buffer supplemented with protease inhibitor tablets (Roche). siRNA experiments were carried out in biological triplicate in a single pair of clonal isogenic MSCs.

For infections, $\varphi \mathrm{NX}$ (Phoenix) cells were transfected with Lipofectamine 2000 (Invitrogen) and either pMMP-puro-vector or pMMP-puro-Flag-HA-NR6A1 plasmid. Nr6a1 cDNA (Isoform 1) was cloned from Dicer knockout MSCs. Viral supernatant was cleared with a $0.45-\mu \mathrm{m}$ syringe filter. Subconfluent MSCs were incubated with viral supernatant at a multiplicity of infection $\leq 1$ and $8 \mu \mathrm{g}$ of polybrene (Sigma) overnight and then selected with $2.5 \mu \mathrm{g}$ of puromycin. Prior to being used for experiments, transduced MSCs were passaged under selection for $\sim 2$ wk. Infections were carried out on two independent clones of Dicer wild-type MSCs, thus representing biological duplicates.

\section{Western blot}

RIPA lysates were diluted twofold with $2 \times$ Laemmli loading buffer with $5 \% \beta$-mercaptoethanol and then boiled for $10 \mathrm{~min}$. Samples were separated in $4 \%-12 \%$ Bis-Tris denaturing polyacrylamide Novex gradient gels (Invitrogen) in an XCell SureLock apparatus (Invitrogen) and transferred to a polyvinylidene fluoride (PVDF) membrane in a Mini Trans-Blot wet transfer apparatus (Bio-Rad). Membranes were blocked with $5 \%$ milk in $1 \times$ Trisbuffered saline with $0.1 \%$ Tween 20 (TBST), incubated overnight at $4{ }^{\circ} \mathrm{C}$ with primary antibody diluted in $5 \%$ milk/TBST, washed three times with $1 \times$ TBST, incubated for $1 \mathrm{~h}$ at room temperature with secondary antibody conjugated to horseradish peroxidase (GE Healthcare Life Sciences), washed three times, incubated briefly with Western Lightning Plus-ECL (PerkinElmer), and visualized on film (Kodak).

The following rabbit polyclonal antibodies were used: antiIGF2BP1 (MBL International), anti-IGF2BP2 (MBL International), anti-IGF2BP3 (MBL International), anti-HMGA2 (Cell Signaling Technology), and anti-p107 (Santa Cruz Biotechnology).

\section{qPCR}

Total RNA was treated with a TURBO DNA-free kit (Ambion). cDNA was generated with oligo-dT primer using a SuperScript III first strand synthesis system (Invitrogen). Gene expression was analyzed with Power SYBR Green (Applied Biosystems).

\section{Microarray analysis}

For MSCs, two clones of Dicer wild-type and their four derivative clones of Dicer knockout cells were grown to confluence in six-well plates, after which total RNA was prepared with QIAzol (Qiagen). A third Dicer wild-type clone, from which no Dicer knockout cells were available, was also analyzed initially but then subsequently excluded as an outlier. For sarcoma samples, Dicer heterozygous and Dicer knockout cells were grown to confluence in T25s, and total RNA was prepared with an RNeasy kit (Qiagen). Biotinylated cRNA was prepared using the Affymetrix GeneChip WT Sense Target Labeling and Control Reagents kit and hybridized to Affymetrix Mouse 430_2 3' arrays (sarcoma cell lines) or Mouse MoEx-1_0-st exon arrays (MSCs) at the BioMicroCenter at MIT.

\section{RNA-seq}

Cloning and sequencing of small RNAs were carried out as described previously (Gurtan et al. 2012; Ravi et al. 2012). mRNA-seq was carried out with total RNA purified with the RNeasy reagent (Qiagen) and DNase-treated with TURBO DNase (Ambion). Samples were prepared for Illumina sequencing at the BioMicroCenter at MIT.

\section{ChIP-seq}

ChIP experiments were carried out as described previously (Rahl et al. 2010). In summary, Dicer wild-type and Dicer knockout MSCs were grown as described above and cross-linked for $10 \mathrm{~min}$ at room temperature by the addition of one-tenth of the volume of $11 \%$ formaldehyde solution $11 \%$ formaldehyde, $50 \mathrm{mM}$ HEPES at $\mathrm{pH} 7.3,100 \mathrm{mM} \mathrm{NaCl}, 1 \mathrm{mM}$ EDTA at $\mathrm{pH}$ 8.0, 0.5 mM EGTA at $\mathrm{pH} 8.0$ ) to the growth medium. Cells were washed twice with PBS, supernatant was aspirated, and the cell pellet was flash-frozen in liquid nitrogen. Frozen cross-linked cells were stored at $-80^{\circ} \mathrm{C}$. For histone mark immunoprecipitation, the following antibodies were used: Histone H3K4me3, Millipore 07-473, lot number DAM1731494 (7.5 $\mu \mathrm{L}$ per ChIP); Histone H3K36me3, Abcam ab9050-100, lot number 136352; Histone H3K27me3, Abcam ab6002-100, lot number 49749. For immunoprecipitation of Flag-HA-NR6A1, anti-HA (Roche, 11867423001) was used.

\section{Acknowledgments}

We thank members of the Sharp laboratory for helpful discussions and experimental assistance. Specifically, we thank Timothy Kelly for generating the doxycycline-inducible Flag-HA-Ago2 construct, and Jesse Zamudio and Mohini Jangi for help with Flag-HA-NR6A1 ChIP. We thank Alla Leshinsky, Richard Cook, and the rest of the Swanson Biotechnology Center at the David $\mathrm{H}$. Koch Institute for Integrative Cancer Research at MIT for Illumina and other sequencing services. We thank Stuart Levine and the staff of the BioMicroCenter at MIT for preparation of RNA-seq and ChIP-seq samples. This work was supported by NIH grant RO1-GM34277 (to P.A.S.), NCI grant PO1-CA42063 (to P.A.S.), RO1-HG002668 (to R.A.Y.), and the NCI Cancer Center Support (core) grant P30-CA14051. A.M.G. acknowledges support from the Leukemia and Lymphoma Society Grant 519809. A.M.G., with contributions from A.R., designed the study. A.M.G., A.R., P.B.R., A.D.B., and C.K.J. performed experiments. P.B.R., under the supervision of R.A.Y., carried out ChIP of 
histone marks. A.D.B. designed and carried out the CLIP-seq experiments. A.M.G., A.R., A.D.B., A.B., and C.A.W. carried out computational analyses. A.B. and C.A.W. performed the informatics for the microarray and sequencing data. A.M.G. wrote the paper. P.A.S. provided supervision and assisted with manuscript preparation. All authors reviewed and approved the manuscript.

\section{References}

Alajez NM, Shi W, Wong D, Lenarduzzi M, Waldron J, Weinreb I, Liu FF. 2012. Lin28b promotes head and neck cancer progression via modulation of the insulin-like growth factor survival pathway. Oncotarget 3: 1641-1652.

Ambros V. 2011. MicroRNAs and developmental timing. Curr Opin Genet Dev 21: 511-517.

Andl T, Murchison EP, Liu F, Zhang Y, Yunta-Gonzalez M, Tobias JW, Andl CD, Seykora JT, Hannon GJ, Millar SE. 2006. The miRNA-processing enzyme dicer is essential for the morphogenesis and maintenance of hair follicles. Curr Biol 16: 1041-1049.

Bartel DP, Chen CZ. 2004. Micromanagers of gene expression: The potentially widespread influence of metazoan microRNAs. Nat Rev Genet 5: 396-400.

Bernstein E, Kim SY, Carmell MA, Murchison EP, Alcorn H, Li MZ, Mills AA, Elledge SJ, Anderson KV, Hannon GJ. 2003. Dicer is essential for mouse development. Nat Genet 35: 215-217.

Boyerinas B, Park SM, Shomron N, Hedegaard MM, Vinther J, Andersen JS, Feig C, Xu J, Burge CB, Peter ME. 2008. Identification of let-7-regulated oncofetal genes. Cancer Res 68: 2587-2591.

Boyerinas B, Park SM, Hau A, Murmann AE, Peter ME. 2010. The role of let-7 in cell differentiation and cancer. Endocr Relat Cancer 17: F19-F36.

Brennecke J, Hipfner DR, Stark A, Russell RB, Cohen SM. 2003. bantam encodes a developmentally regulated microRNA that controls cell proliferation and regulates the proapoptotic gene hid in Drosophila. Cell 113: 25-36.

Calabrese JM, Seila AC, Yeo GW, Sharp PA. 2007. RNA sequence analysis defines Dicer's role in mouse embryonic stem cells. Proc Natl Acad Sci 104: 18097-18102.

Chi SW, Zang JB, Mele A, Darnell RB. 2009. Argonaute HITSCLIP decodes microRNA-mRNA interaction maps. Nature 460: 479-486.

Chung AC, Katz D, Pereira FA, Jackson KJ, DeMayo FJ, Cooney AJ, O'Malley BW. 2001. Loss of orphan receptor germ cell nuclear factor function results in ectopic development of the tail bud and a novel posterior truncation. Mol Cell Biol 21: 663-677.

Colas AR, McKeithan WL, Cunningham TJ, Bushway PJ, Garmire LX, Duester G, Subramaniam S, Mercola M. 2012. Wholegenome microRNA screening identifies let-7 and mir-18 as regulators of germ layer formation during early embryogenesis. Genes Dev 26: 2567-2579.

David R, Joos TO, Dreyer C. 1998. Anteroposterior patterning and organogenesis of Xenopus laevis require a correct dose of germ cell nuclear factor (xGCNF). Mech Dev 79: 137-152.

Dugas JC, Cuellar TL, Scholze A, Ason B, Ibrahim A, Emery B, Zamanian JL, Foo LC, McManus MT, Barres BA. 2010. Dicer1 and miR-219 Are required for normal oligodendrocyte differentiation and myelination. Neuron 65: 597-611.

Ebert MS, Sharp PA. 2012. Roles for microRNAs in conferring robustness to biological processes. Cell 149: 515-524.

Fedele M, Visone R, De Martino I, Troncone G, Palmieri D, Battista S, Ciarmiello A, Pallante P, Arra C, Melillo RM, et al. 2006. HMGA2 induces pituitary tumorigenesis by enhancing E2F1 activity. Cancer Cell 9: 459-471.
Frost RJ, Olson EN. 2011. Control of glucose homeostasis and insulin sensitivity by the Let-7 family of microRNAs. Proc Natl Acad Sci 108: 21075-21080.

Fuhrmann G, Chung AC, Jackson KJ, Hummelke G, Baniahmad A, Sutter J, Sylvester I, Scholer HR, Cooney AJ. 2001. Mouse germline restriction of Oct4 expression by germ cell nuclear factor. Dev Cell 1: 377-387.

Ghildiyal M, Zamore PD. 2009. Small silencing RNAs: An expanding universe. Nat Rev Genet 10: 94-108.

Gu P, LeMenuet D, Chung AC, Mancini M, Wheeler DA, Cooney AJ. 2005. Orphan nuclear receptor GCNF is required for the repression of pluripotency genes during retinoic acidinduced embryonic stem cell differentiation. Mol Cell Biol 25: 8507-8519.

Gurtan AM, Sharp PA. 2013. The role of miRNAs in regulating gene expression networks. I Mol Biol. doi: 10.1016/j.jmb. 2013.03.007.

Gurtan AM, Lu V, Bhutkar A, Sharp PA. 2012. In vivo structurefunction analysis of human Dicer reveals directional processing of precursor miRNAs. RNA 18: 1116-1122.

Hammell CM, Karp X, Ambros V. 2009. A feedback circuit involving let-7-family miRNAs and DAF-12 integrates environmental signals and developmental timing in Caenorhabditis elegans. Proc Natl Acad Sci 106: 18668-18673.

Hansen TV, Hammer NA, Nielsen J, Madsen M, Dalbaeck C, Wewer UM, Christiansen J, Nielsen FC. 2004. Dwarfism and impaired gut development in insulin-like growth factor II mRNA-binding protein 1-deficient mice. Mol Cell Biol 24: 4448-4464.

Harfe BD, McManus MT, Mansfield JH, Hornstein E, Tabin CJ. 2005. The RNaseIII enzyme Dicer is required for morphogenesis but not patterning of the vertebrate limb. Proc Nat1 Acad Sci 102: 10898-10903.

Herranz H, Cohen SM. 2010. MicroRNAs and gene regulatory networks: Managing the impact of noise in biological systems. Genes Dev 24: 1339-1344.

Hirning-Folz U, Wilda M, Rippe V, Bullerdiek J, Hameister H. 1998. The expression pattern of the Hmgic gene during development. Genes Chromosomes Cancer 23: 350-357.

Irie N, Kuratani S. 2011. Comparative transcriptome analysis reveals vertebrate phylotypic period during organogenesis. Nat Commun 2: 248.

Johnson SM, Grosshans H, Shingara J, Byrom M, Jarvis R, Cheng A, Labourier E, Reinert KL, Brown D, Slack FJ. 2005. RAS is regulated by the let-7 microRNA family. Cell 120: 635-647.

Johnson CD, Esquela-Kerscher A, Stefani G, Byrom M, Kelnar K, Ovcharenko D, Wilson M, Wang X, Shelton J, Shingara J, et al. 2007. The let-7 microRNA represses cell proliferation pathways in human cells. Cancer Res 67: 7713-7722.

Kanellopoulou C, Muljo SA, Kung AL, Ganesan S, Drapkin R, Jenuwein T, Livingston DM, Rajewsky K. 2005. Dicer-deficient mouse embryonic stem cells are defective in differentiation and centromeric silencing. Genes Dev 19: 489-501.

Kim J, Woo AJ, Chu J, Snow JW, Fujiwara Y, Kim CG, Cantor AB, Orkin SH. 2010. A Myc network accounts for similarities between embryonic stem and cancer cell transcription programs. Cell 143: 313-324.

Koralov SB, Muljo SA, Galler GR, Krek A, Chakraborty T, Kanellopoulou C, Jensen K, Cobb BS, Merkenschlager M, Rajewsky N, et al. 2008. Dicer ablation affects antibody diversity and cell survival in the B lymphocyte lineage. Cell 132: $860-874$.

Lee YS, Dutta A. 2007. The tumor suppressor microRNA let-7 represses the HMGA2 oncogene. Genes Dev 21: 10251030. 
Lee RC, Feinbaum RL, Ambros V. 1993. The C. elegans heterochronic gene lin-4 encodes small RNAs with antisense complementarity to lin-14. Cell 75: 843-854.

Leung AK, Young AG, Bhutkar A, Zheng GX, Bosson AD, Nielsen CB, Sharp PA. 2011. Genome-wide identification of Ago2 binding sites from mouse embryonic stem cells with and without mature microRNAs. Nat Struct Mol Biol 18: 237-244.

Li Z, Gilbert JA, Zhang Y, Zhang M, Qiu Q, Ramanujan K, Shavlakadze T, Eash JK, Scaramozza A, Goddeeris MM, et al. 2012. An HMGA2-IGF2BP2 axis regulates myoblast proliferation and myogenesis. Dev Cell 23: 1176-1188.

Marson A, Levine SS, Cole MF, Frampton GM, Brambrink $\mathrm{T}$, Johnstone S, Guenther MG, Johnston WK, Wernig M, Newman J, et al. 2008. Connecting microRNA genes to the core transcriptional regulatory circuitry of embryonic stem cells. Cell 134: $521-533$.

Mayr C, Bartel DP. 2009. Widespread shortening of 3'UTRs by alternative cleavage and polyadenylation activates oncogenes in cancer cells. Cell 138: 673-684.

Mayr C, Hemann MT, Bartel DP. 2007. Disrupting the pairing between let-7 and Hmga2 enhances oncogenic transformation. Science 315: 1576-1579.

Melton C, Judson RL, Blelloch R. 2010. Opposing microRNA families regulate self-renewal in mouse embryonic stem cells. Nature 463: 621-626.

Muljo SA, Ansel KM, Kanellopoulou C, Livingston DM, Rao A, Rajewsky K. 2005. Aberrant $\mathrm{T}$ cell differentiation in the absence of Dicer. J Exp Med 202: 261-269.

Murchison EP, Partridge JF, Tam OH, Cheloufi S, Hannon GJ. 2005. Characterization of Dicer-deficient murine embryonic stem cells. Proc Natl Acad Sci 102: 12135-12140.

Nimmo RA, Slack FJ. 2009. An elegant miRror: MicroRNAs in stem cells, developmental timing and cancer. Chromosoma 118: $405-418$.

O'Rourke JR, Georges SA, Seay HR, Tapscott SJ, McManus MT, Goldhamer DJ, Swanson MS, Harfe BD. 2007. Essential role for Dicer during skeletal muscle development. Dev Biol 311: 359-368.

Peeper DS, Shvarts A, Brummelkamp T, Douma S, Koh EY, Daley GQ, Bernards R. 2002. A functional screen identifies hDRIL1 as an oncogene that rescues RAS-induced senescence. Nat Cell Biol 4: 148-153.

Rahl PB, Lin CY, Seila AC, Flynn RA, McCuine S, Burge CB, Sharp PA, Young RA. 2010. c-Myc regulates transcriptional pause release. Cell 141: 432-445.

Ravi A, Gurtan AM, Kumar MS, Bhutkar A, Chin C, Lu V, Lees JA, Jacks T, Sharp PA. 2012. Proliferation and tumorigenesis of a murine sarcoma cell line in the absence of DICER1. Cancer Cell 21: 848-855.

Reinhart BJ, Slack FJ, Basson M, Pasquinelli AE, Bettinger JC, Rougvie AE, Horvitz HR, Ruvkun G. 2000. The 21-nucleotide let-7 RNA regulates developmental timing in Caenorhabditis elegans. Nature 403: 901-906.

Schulman BR, Esquela-Kerscher A, Slack FJ. 2005. Reciprocal expression of lin-41 and the microRNAs let-7 and mir-125 during mouse embryogenesis. Dev Dyn 234: 1046-1054.

Shapiro JS, Langlois RA, Pham AM, Tenoever BR. 2012. Evidence for a cytoplasmic microprocessor of pri-miRNAs. RNA 18: $1338-1346$.

Taher L, Collette NM, Murugesh D, Maxwell E, Ovcharenko I, Loots GG. 2011. Global gene expression analysis of murine limb development. PLOS ONE 6: e28358.

Takebe A, Era T, Okada M, Martin Jakt L, Kuroda Y, Nishikawa S. 2006. Microarray analysis of PDGFR $\alpha^{+}$populations in ES cell differentiation culture identifies genes involved in differ- entiation of mesoderm and mesenchyme including ARID3b that is essential for development of embryonic mesenchymal cells. Dev Biol 293: 25-37.

Tessier CR, Doyle GA, Clark BA, Pitot HC, Ross J. 2004. Mammary tumor induction in transgenic mice expressing an RNA-binding protein. Cancer Res 64: 209-214.

Trang P, Medina PP, Wiggins JF, Ruffino L, Kelnar K, Omotola M, Homer R, Brown D, Bader AG, Weidhaas JB, et al. 2010. Regression of murine lung tumors by the let-7 microRNA. Oncogene 29: 1580-1587.

Viswanathan SR, Daley GQ. 2010. Lin28: A microRNA regulator with a macro role. Cell 140: 445-449.

Viswanathan SR, Powers JT, Einhorn W, Hoshida Y, Ng TL, Toffanin S, O'Sullivan M, Lu J, Phillips LA, Lockhart VL, et al. 2009. Lin 28 promotes transformation and is associated with advanced human malignancies. Nat Genet 41: 843-848.

Vitobello A, Ferretti E, Lampe X, Vilain N, Ducret S, Ori M, Spetz JF, Selleri L, Rijli FM. 2011. Hox and Pbx factors control retinoic acid synthesis during hindbrain segmentation. Dev Cell 20: 469-482.

Wang Y, Medvid R, Melton C, Jaenisch R, Blelloch R. 2007. DGCR8 is essential for microRNA biogenesis and silencing of embryonic stem cell self-renewal. Nat Genet 39: 380-385.

Wong DJ, Liu H, Ridky TW, Cassarino D, Segal E, Chang HY. 2008. Module map of stem cell genes guides creation of epithelial cancer stem cells. Cell Stem Cell 2: 333-344.

Wu C, Orozco C, Boyer J, Leglise M, Goodale I, Batalov S, Hodge CL, Haase J, Janes J, Huss JW III, et al. 2009. BioGPS: An extensible and customizable portal for querying and organizing gene annotation resources. Genome Biol 10: R130.

Wu C, Macleod I, Su AI. 2013. BioGPS and MyGene.info: Organizing online, gene-centric information. Nucleic Acids Res 41: D561-D565.

Yan ZH, Medvedev A, Hirose T, Gotoh H, Jetten AM. 1997. Characterization of the response element and DNA binding properties of the nuclear orphan receptor germ cell nuclear factor/retinoid receptor-related testis-associated receptor. J Biol Chem 272: 10565-10572.

Yi R, O'Carroll D, Pasolli HA, Zhang Z, Dietrich FS, Tarakhovsky A, Fuchs E. 2006. Morphogenesis in skin is governed by discrete sets of differentially expressed microRNAs. Nat Genet 38: 356-362.

Zaidi MR, Okada Y, Chada KK. 2006. Misexpression of fulllength HMGA2 induces benign mesenchymal tumors in mice. Cancer Res 66: 7453-7459.

Zheng $\mathrm{H}$, Ying $\mathrm{H}$, Wiedemeyer R, Yan $\mathrm{H}$, Quayle SN, Ivanova EV, Paik JH, Zhang H, Xiao Y, Perry SR, et al. 2010. PLAGL2 regulates Wnt signaling to impede differentiation in neural stem cells and gliomas. Cancer Cell 17: 497-509.

Zhou X, Benson KF, Ashar HR, Chada K. 1995. Mutation responsible for the mouse pygmy phenotype in the developmentally regulated factor HMGI-C. Nature 376: 771-774.

Zhu H, Shah S, Shyh-Chang N, Shinoda G, Einhorn WS, Viswanathan SR, Takeuchi A, Grasemann C, Rinn JL, Lopez MF, et al. 2010. Lin28a transgenic mice manifest size and puberty phenotypes identified in human genetic association studies. Nat Genet 42: 626-630.

Zhu H, Shyh-Chang N, Segre AV, Shinoda G, Shah SP, Einhorn WS, Takeuchi A, Engreitz JM, Hagan JP, Kharas MG, et al. 2011. The Lin28/let-7 axis regulates glucose metabolism. Cell 147: 81-94. 


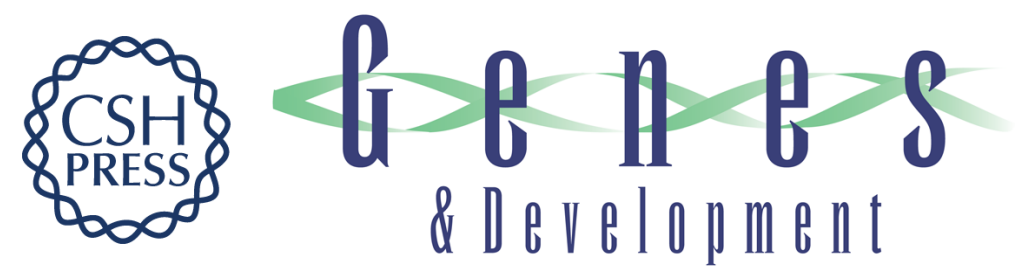

\section{Let-7 represses Nr6a1 and a mid-gestation developmental program in adult fibroblasts}

Allan M. Gurtan, Arvind Ravi, Peter B. Rahl, et al.

Genes Dev. 2013, 27:

Access the most recent version at doi:10.1101/gad.215376.113

Supplemental http://genesdev.cshlp.org/content/suppl/2013/04/29/27.8.941.DC1
Material

References This article cites 68 articles, 22 of which can be accessed free at: http://genesdev.cshlp.org/content/27/8/941.full.html\#ref-list-1

License

Email Alerting Receive free email alerts when new articles cite this article - sign up in the box at the top Service right corner of the article or click here.

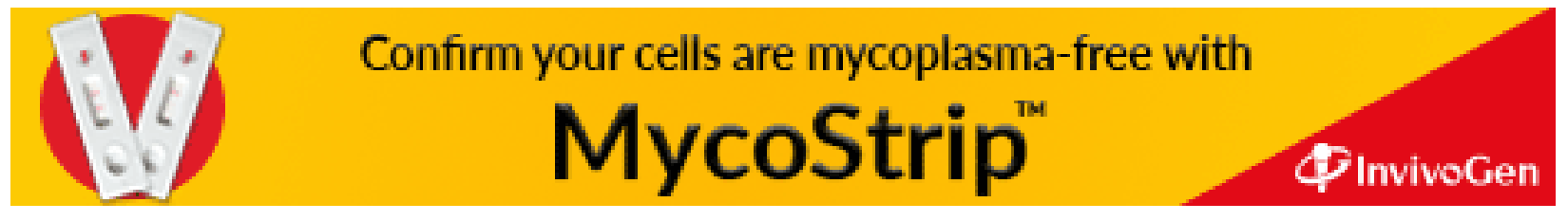

